\section{In the Spotlight}

\author{
Maria Rosa Maduro, PhD
}

\section{Reproductive Sciences on the Forefront of Endometriosis News}

Endometriosis is a gynecologic pathology characterized by the growth and proliferation of endometrial cells outside the uterine cavity. The disease, which is estimated to afflict $4 \%$ to $10 \%$ of women worldwide, is often associated with severe abdominal pain and has also been linked with infertility.

Although much research has been done on endometriosis, the etiology and mechanisms of action of the disease remain unclear and poorly understood. This lack of knowledge has hindered the development of new and more effective ways of preventing, diagnosing, and managing the disease. Therefore, researchers continue to investigate the causes, the molecular pathways of progression, and the pathophysiologic characteristics of endometriosis to, step by step, add pieces to the puzzle in order to be able to better understand and overcome this disease.

Accordingly, Reproductive Sciences has been committed to publish relevant advances on endometriosis research, ${ }^{1-4}$ and particularly in the present issue of Reproductive Sciences, 3 independent groups report their findings on different aspects of the pathology.

Appleyard et $\mathrm{al}^{5}$ describe how stress management affects the pathophysiology of endometriosis in an animal model. This group had previously demonstrated that stress had a detrimental effect on endometriosis. This time, using rats with endometriosis exposed to a controllable or noncontrollable swim stress protocol, Appleyard and colleagues show that the animals subjected to uncontrollable stress presented with increased number and size of endometriotic cysts, higher levels of corticosterone, and anxiety than their counterparts exposed to controllable stress or no stress at all. The former group of animals also showed more colonic damage and uterine infiltration, as well as increased colonic and uterine motility compared to the other 2 control groups. These findings evidence the impact that stress controllability has on endometriosis pathophysiology and indicate that this behavior should be considered as a therapeutic strategy.

Fanasiak et al, ${ }^{6}$ on the other hand, focused their research on the molecular players involved in endometriosis progression. More specifically, the authors were interested in understanding the involvement of a regulator of mucosal immunity, known as C-X-C ligand 13 (CXCL13), in the disease. Knowing that CXCL13 is secreted by the human endometrial epithelium,
Fanasiak and colleagues aimed to characterize its cyclic expression in healthy and endometrial participants, using immunohistochemistry and reverse transcription-polymerase chain reaction as their tools. In addition, the authors determined eutopic endometrial expression in normal Rhesus macaques and animals with advanced endometriosis. Their results showed that in humans, expression of CXCL13 was minimal in the proliferative phase and achieved its high in the secretory phase. In both humans and Rhesus macaques, expression of CXCL13 was significantly increased in the proliferative phase of patients presenting the disease. The fact that expression of CXCL13 was concordantly increased in a specific stage of the disease cross-species highlights the relevance of this expression. These findings not only suggest a pathophysiologic role for CXCL13 in endometriosis but also indicate that this molecule may be useful as a biomarker for the disease.

Finally, Montaño et $\mathrm{al}^{7}$ aimed to investigate whether the molecular pathways commonly associated with altered cell proliferation in ectopic endometrium also play a role in eutopic endometrium during endometriosis. The authors obtained eutopic endometrium from 35 women with endometriosis and 25 fertile eumenorrheic women and analyzed in situ expression of stem cell factor (SCF)/c-kit, Ki67, Akt, phosphorylated Akt (pAkt), GSK3 $\beta$, and phosphorylated GSK3 $\beta$ (pGSK3 $\beta$ ), during the menstrual cycle, through immunohistochemistry. The results obtained by Montaño and colleagues showed that expression of Ki67 and SCF was higher in endometriosis samples than in the control tissue and greater in the secretory phase rather than the proliferative phase of the cycle in endometriosis. For expression of c-kit, the authors observed an increase in endometriosis independent of the phase. Although expression of Akt and GSK3 $\beta$ was similar in all samples and cycle phases, pAkt and pGSK $3 b$ were overexpressed in the secretory phase in endometriosis samples. Altogether, the molecular characterization of cell proliferation pathways and stem cell markers performed by Montaño and colleagues indicate that there is an increase expression of SCF/c-kit and an impaired signaling by, most likely, the perpetuation of Akt and GSK3 $\beta$ phosphorylation in eutopic endometriosis, which results in maintained epithelial cell proliferation in the secretory phase of the menstrual cycle. These findings help better understand the molecular events supporting endometriosis progression. 


\section{References}

1. Stanic AK, Kim M, Styer AK, Rueda BR. Dendritic cells attenuate the early establishment of endometriosis-like lesions in a murine model. Reprod Sci. 2014;21(10):1228-1236.

2. Naqvi H, Ilagan Y, Krikun G, Taylor HS. Altered genomewide methylation in endometriosis. Reprod Sci. 2014;21(10): 1237-1243.

3. Governini L, Carrarelli P, Rocha AL, et al. FOXL2 in human endometrium: hyperexpressed in endometriosis. Reprod Sci. 2014;21(10):1249-1255.

4. Carr B, Dmowski WP, O'Brien C, et al. Elagolix, an oral GnRH antagonist versus subcutaneous depot medroxyprogesterone acetate for the treatment of endometriosis: effects on bone mineral density. Reprod Sci. 2014;21(11):1341-1351.
5. Appleyard CB, Cruz ML, Hernández S, Thompson KJ, Bayona M, Flores I. Stress management affects outcomes in the pathophysiology of an endometriosis model [published online ahead of print July 11, 2014]. Reprod Sci. doi: 10.1177/1933719114542022.

6. Fanasiak J, Burns K, Slayden O, et al. Endometrial CXCL13 Expression is cycle regulated in humans and aberrantly expressed in humans and rhesus macaques with endometriosis [published online ahead of print July 16, 2014]. Reprod Sci. doi: 10.1177/ 1933719114542011.

7. Franco-Murillo Y, Miranda-Rodríguez JA, Rendón-Huerta E, et al. Unremitting cell proliferation in the secretory phase of eutopic endometriosis: Involvement of pAkt and pGSK3 $\beta$ [published online ahead of print September 6, 2014]. Reprod Sci. doi: $10.1177 / 1933719114549843$. 